\title{
The Effect of the Aqueous Extract of Bidens Pilosa L. on Androgen Deficiency Dry Eye in Rats
}

\author{
Chuanwei Zhang ${ }^{a}$ Kai Li ${ }^{a}$ Zichao Yang ${ }^{a}$ Yuliang Wang a Haipeng Sib \\ aDepartment of Ophthalmology, the First Affiliated Hospital of Nanjing University of Chinese Medicine, \\ Nanjing, ${ }^{b}$ Department of Pathology, the First Affiliated Hospital of Nanjing University of Chinese \\ Medicine, Nanjing, China
}

\section{Key Words}

Androgen deficiency dry eye $\bullet$ Sex steroids $\bullet$ Bidens pilosa L. $\bullet$ Experimental models

\begin{abstract}
Background/Aims: Bidens pilosa L. (Bp) is widely distributed in China and has been widely used as a traditional Chinese medicine. The aim of this study was to examine the effect of the extract of $B p$ on androgen deficiency dry eye and determine its possible mechanisms. Methods: Twenty-four rats were randomly divided into four groups: Group Con (control), Group Sal (physiological saline), Group Fin (oral finasteride), and Group Bp (oral finasteride and Bp). The dry eye model was established in group Fin and group Bp. Aqueous tear quantity was measured with phenol red-impregnated cotton threads with anesthesia. Tear film breakup time (BUT) and corneal epithelial damage were evaluated by fluorescein staining. Animals were sacrificed at 28 days, and ocular tissues (lacrimal gland and cornea) were evaluated with light microscopy; gene microarray analysis for inflammatory cytokines and Western blot were also performed. Results: Finasteride administration effectively induced dry eye in rats by 14 days after administration. Group Fin rats had significantly higher fluorescein staining scores and lower aqueous tear quantity and BUT than the group Con rats, and notable inflammatory cell infiltrates were observed in the lacrimal gland of group Fin rats. The fluorescein staining score, aqueous tear quantity and BUT significantly improved with Bp treatment in the group Bp rats, and the structures of the lacrimal gland were well maintained without significant lymphocyte infiltration. Cytokine antibody array data identified the cytokines B7-2/Cd86, IL-1 $\beta$, IL-4, IL-6, IL-10, MMP-8, FasL, TNF- $\alpha$ and TIMP-1 as candidates for validation by Western blot. Expression levels of pro-inflammatory cytokines, including IL-1 $\beta$, IL- 6 , and TNF- $\alpha$, in group Fin were upregulated compared with group Con. Levels of anti-inflammatory cytokines, such as IL-4 and IL-10, in group Fin were also upregulated compared with those in group Con. Compared with group Fin, IL-1 $\beta$, FasL, and TNF- $\alpha$ were significantly decreased in group $B p$. Conclusion: The extract of $B p$ appears to be effective for the treatment of androgen deficiency dry eye in rats by improving aqueous tear quantity, maintaining tear film stability, and inhibiting the inflammation of the lacrimal gland.




\section{Cellular Physiology Cell Physiol Biochem 2016;39:266-277 and Biochemistry Published online: June 24, $2016 \quad \begin{aligned} & \text { DOI: 10.1159/000445622 } 2016 \text { The Author(s). Published by S. Karger AG, Basel } \\ & \text { www.karger.com/cpb }\end{aligned}$ \\ Zhang et al.: The Effect of Bidens Pilosa L. on Dry Eye}

\section{Introduction}

Dry eye is a common ocular surface disease characterized by unstable tear film, ocular surface epithelial disease and inflammation, lacrimal gland inflammation, and secretory dysfunction [1]. The major etiological causes of dry eye can be classified into two groups: aqueous deficiency due to insufficient lacrimal tear secretion and excessive evaporation [2], and aqueous deficiency or excessive evaporation caused by tear hyperosmolarity. These changes can activate the MAPK and NF- $\mathrm{KB}$ signaling pathways, which results in increased concentrations of proinflammatory mediators and inflammatory cytokine expression [3]. The prevalence of dry eye was reported to range from $5 \%$ to $35 \%$, and the condition was more prevalent in women and could be increased with age [4]. The epidemiological studies indicated that the prevalence of dry eye is approximately $7 \%$ in women and $4 \%$ in men in the US amongst those $>50$ years of age [5]. The decline of androgen levels was proven to be one main cause of dry eye, as androgen plays a major role in the regulation of the lacrimal gland secretary functions [6]. Therefore, androgen replacement therapy (ART) is currently considered the only effective therapy for dry eye caused by a gonadal hormone imbalance. However, the long-term treatment of ART would inevitably lead to some adverse effects, and accordingly, it is necessary to search for new safe and effective agents to treat dry eye.

Bidens pilosa L. $(B p)$ is an easy-to-grow herb that is widely distributed in almost all tropical and subtropical region countries around the world [7]. As a folk medicine, $B p$ has been used in various medications. Recent studies revealed that $B p$ possesses various promising pharmacological effects, such as anti-allergic [8], anti-ulcer [9], anti-inflammatory [10] and immunomodulatory properties $[11,12]$. Particularly, $B p$ has attracted much interest due to its proven pharmacologic safety and its anti-inflammatory activities through inhibiting the production of pro-inflammatory cytokines. Our previous studies showed that $B p$ had the potential to serve as a therapeutic agent for dry eye $[13,14]$; however, the mechanism of action and whether $B p$ is useful for inhibiting inflammation in dry eye remain unclear. Therefore, the present study was designed to evaluate the therapeutic effect of $B p$ on dry eye and explore its possible underlying mechanism of action in a rat model of androgen deficiency.

\section{Materials and Methods}

\section{Experimental animals}

Healthy female Wistar rats (7 months old, weighing 180 - $250 \mathrm{~g}$ ) were obtained from the animal experimental center of Nanjing Medical University. The animals were housed in ambient conditions of room temperature $25 \pm 2^{\circ} \mathrm{C}$, relative humidity $35 \% \pm 5 \%$, an alternating 12-hour light-dark cycle and food and water ad libitum. All experimental protocols were approved by the Animal Care and Use Committee of Nanjing University of Chinese Medicine.

Animal models

Before the experiment, all animals were subjected to the analysis of the anterior segment of the eyes, and only the rats with normal eyes were included in this study. Ultimately, 24 rats were selected and randomly divided into four groups (A, B, C and D), with each group consisting of 6 rats. Group A served as the normal control without any treatment (group Con), group B received saline gastric perfusion (group Sal), group $C$ received finasteride gastric perfusion (group Fin), and group D simultaneously received $B p$ extract and finasteride gastric perfusion (group $B p$ ). All animals were analyzed for tear flow, break up time and fluorescein staining assessment. Day ' 0 ' served as the baseline as well as the control for the respective groups for 28 days.

Collection and preparation of water extract of $B P$

The herbal plant of Bp was purchased from the Fujian herbal medicines planting base (Fuzhou, China). The authenticity of the plant was confirmed by Dr. Hua Yongqing, a botanist of the Department of Pharmaceutical Sciences at Nanjing University of Chinese Medicine (Nanjing, China). For the present study, the aqueous extract of $B p$ was prepared according to the traditional method [15]. First, leaves of 


\section{Cellular Physiology Cell Physiol Biochem 2016;39:266-277

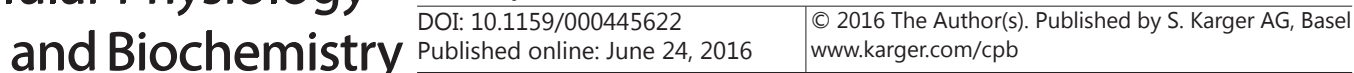 \\ Zhang et al.: The Effect of Bidens Pilosa L. on Dry Eye}

$B p$ were dried in an oven at $60^{\circ} \mathrm{C}$ for 72 hours and ground with a mortar and pestle. Next, two hundred grams of dried leaves of $B p$ were boiled in one liter of water for twenty minutes. Finally, the obtained liquid was freeze-dried to yield $38 \mathrm{~g}$ of a brownish powder. A stock solution of $100 \mathrm{mg} / \mathrm{ml}$ in distilled water was prepared daily because of the high instability of $B p$ 's components.

\section{Administration method}

Group Sal: daily administration by gavage of $0.5 \mathrm{ml}$ per 100 grams of body weight of saline once a day for 4 weeks.

Group Fin: finasteride $(1.16 \mathrm{mg} / \mathrm{kg} / \mathrm{d}$ ) (Msd International Gmbh Co. Ltd, Sabana Hoyos, Arecibo, Puerto Rico) was orally administered to all the rats once a day for 4 weeks.

Group $B p$ : in addition to oral finasteride as group Fin, $0.5 \mathrm{ml}$ per 100 grams of body weight of water extract of $B p$ was orally administered to all the rats once a day for 4 weeks.

Treatments in our current study were administered by gavage.

\section{Tear secretion and stability of tear film examinations}

The phenol red thread test, tear film breakup time (BUT) and fluorescein staining were performed to determine tear secretion and the stability of the tear film. The time points for measuring were day $0,7,14$, 21 and 28. Day ' 0 ' served as the baseline as well as the control for their respective groups. Tear secretion was measured in lightly anesthetized rats using phenol red-impregnated cotton threads (Tianjin Jingming New Technological Development Co., Ltd, Tianjin, China). The threads were held with microforceps and applied to the lateral canthus of both eyes for $60 \mathrm{~s}$. Wetting of the thread (which turns red in contact with tears) was measured in millimeters. One drop of $0.5 \%$ fluorescein solution was applied to the conjunctival sac. BUT and corneal staining were observed by slit lamp microscopy (Topcon SL-D7, Tokyo, Japan) with a cobalt blue filter. BUT was initially evaluated by waiting for the natural blink response of the rat, which was recorded three times, and the mean of the measurements was then calculated. Punctate staining was recorded after BUT examination, and the staining indicated the damage of the corneal epithelium. The staining grade was classified using the following standards: grade 0 : no staining; grade 1: 1/8 was stained; grade 2:1/4 was stained; grade 3:1/2 was stained; grade 4: > 1/2 was stained.

\section{Determination of testosterone (T)}

Blood was collected from the caudal vein of rats. After collection, the blood samples were centrifuged at $3000 \mathrm{~g}$ for $10 \mathrm{~min}$ at $4^{\circ} \mathrm{C}$, and aliquots of plasma were stored at $-20^{\circ} \mathrm{C}$ until assay. Serum testosterone concentrations were measured by radioimmunoassay using commercial kits (Diagnostic Products Co, Los Angeles, CA, USA) following the manufacturer's instructions. The time points for measuring were day 0,14 and 28 .

\section{Light microscopy examination}

After humane euthanasia, a strip of the lacrimal gland and cornea measuring approximately $5 \mathrm{~mm}$ in width vertically across the globe were removed from all rats and fixed in $10 \%$ formalin. After dehydration, the specimens were embedded in paraffin, cross-sectioned, and stained with hematoxylin and eosin (H\&E). The morphology of the lacrimal gland and cornea was observed under a light microscope using $40 \times$ and $10 \times$ magnification.

\section{Protein extraction and cytokine antibody array detection}

Raybio $^{\circledast}$ Rat Cytokine Antibody Arrays G Series 2 were performed according to the manufacturer's protocol (RayBiotech, Inc., Norcross, GA, USA) on tissues from individual animals. Total protein was extracted from the lacrimal gland and cornea using a tissue protein extraction reagent (Kangchen Biotech, Shanghai, China). The protein concentration was determined for each sample using the BCA method. Rat cytokine antibody arrays were used to detect the expression of 35 cytokines in the lacrimal gland and cornea from four groups. The signal from the membrane was detected with a chemiluminescence imaging system. By comparing the signal intensities, the relative expression levels of cytokines were calculated. The intensities of signals could be quantified by densitometry. Positive controls were used to normalize the results from the different membranes being compared. Compared with the control group, the expression of the corresponding cytokines was increased or decreased, such that a ratio greater than 1.3 was high expression and a ratio lower than 0.77 was low expression. 


\section{Cellular Physiology Cell Physiol Biochem 2016;39:266-277

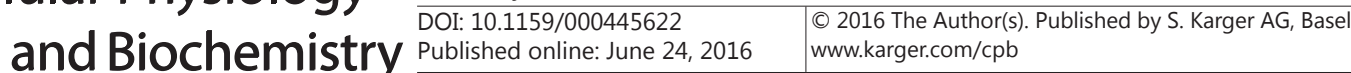 \\ Zhang et al.: The Effect of Bidens Pilosa L. on Dry Eye}

Table 1. Position of 35 rat inflammatory factors in the antibody-based protein microarray. Abbreviations: POS, positive; NEG, negative; $\beta$-NGF, $\beta$ Nerve growth factor; CINC, cytokine-induced neutrophil chemoattractant; CNTF, ciliary neurotrophic factor; GM-CSF, granulocyte-macrophage colony stimulating factor; ICAM1 , intercellular adhesion molecule-1; IFN- $\gamma$, interferon- $\gamma$; IL, interleukin; MCP, monocyte chemoattractant protein; MIP, macrophage inflammatory protein; MMP, matrix metalloproteinase; PDGF, platelet-derived growth factor; RAGE, receptor for advanced glycation end products; TIMP, tissue-specific inhibitor of metalloproteinase; TNF, tumor necrosis factor; VEGF, vascular endothelial growth factor

\begin{tabular}{|c|c|c|c|c|c|c|c|c|c|c|c|c|}
\hline & $\mathrm{A}$ & $\mathrm{B}$ & $\mathrm{C}$ & $\mathrm{D}$ & $\mathrm{E}$ & $\mathrm{F}$ & $\mathrm{G}$ & $\mathrm{H}$ & $\mathrm{I}$ & $\mathrm{J}$ & $\mathrm{K}$ & $\mathrm{L}$ \\
\hline 1 & POS & POS & NEG & NEG & Activin A & Agrin & $\mathrm{B} 7-2 / \mathrm{Cd} 86$ & $\beta$-NGF & CINC-1 & CINC- $2 \alpha$ & $\mathrm{CNNC}-3$ & CNTF \\
\hline 2 & POS & POS & NEG & NEG & Activin A & Agrin & $\mathrm{B} 7-2 / \mathrm{Cd} 86$ & $\beta-\mathrm{NGF}$ & CINC-1 & CINC- $2 \alpha$ & CINC-3 & CNTF \\
\hline 3 & Fas Ligand & Fractalkine & GM- CSF & ICAM-1 & IFN- $\gamma$ & IL-1 $\alpha$ & $\mathbb{I L}-1 \beta$ & IL-1 R6 & $\mathbb{I L}-2$ & IL-4 & IL -6 & $\mathbb{I L}-10$ \\
\hline 4 & Fas Ligand & Fractalkine & GM- CSF & ICAM-1 & IFN- $\gamma$ & IL- $1 \alpha$ & IL- $1 \beta$ & IL-1 R6 & $\mathrm{IL}-2$ & IL-4 & $\mathrm{IL}-6$ & $\mathrm{IL}-10$ \\
\hline 5 & IL-13 & Leptin & LIX & L-Selectin & MCP-1 & MIP-3 $\alpha$ & MMP-8 & PDGF-AA & Prolactin R & RAGE & $\begin{array}{c}\text { Thymus } \\
\text { Chemokine-1 }\end{array}$ & TIMP-1 \\
\hline 6 & $\mathrm{IL}-13$ & Leptin & LIX & L-Selectin & MCP-1 & MIP-3 $\alpha$ & MMP-8 & PDGF-AA & Prolactin R & RAGE & $\begin{array}{c}\text { Thymus } \\
\text { Chemokine-1 }\end{array}$ & TIMP-1 \\
\hline 7 & TNF- $\alpha$ & VEGF & BLANK & BLANK & BLANK & BLANK & BLANK & BLANK & BLANK & BLANK & BLANK & POS \\
\hline 8 & TNF- $\alpha$ & VEGF & BLANK & BLANK & BLANK & BLANK & BLANK & BLANK & BLANK & BLANK & BLANK & POS \\
\hline
\end{tabular}

Western blot analysis

Based on the protein microarray results, only highly expressed cytokines were selected for further investigation with Western blot. Lacrimal glands and corneas were removed from four groups of rats. Tissues used for Western blot analysis were homogenized in lysis buffer (10 mM Tris, pH 8.0, 1 mM EDTA, and a proteinase inhibitor cocktail) and stored at $-80^{\circ} \mathrm{C}$ for Western blot analysis. Tissue samples were diluted in sodium dodecyl sulfate (SDS)-polyacrylamide gel electrophoresis sample buffer. All samples and molecular weight standards were boiled for 5 min prior to being loaded onto 4 to $15 \%$ polyacrylamide gradient gels (Bio-Rad, Hercules, CA, USA). The gels were run for $30 \mathrm{~min}$ at $80 \mathrm{~V}$ and $90 \mathrm{~min}$ at $120 \mathrm{~V}$, washed in transfer buffer and electroblotted onto nitrocellulose membranes for $2 \mathrm{~h}$ at $100 \mathrm{~mA}$. The blots were blocked overnight at $4{ }^{\circ} \mathrm{C}$ with $3 \%$ milk in Tris-buffered saline (TBS) and incubated for $2 \mathrm{~h}$ at room temperature with the corresponding antibody. Following three rinses, the blots were incubated with horseradish peroxidase (HRP)-conjugated rabbit anti-goat immunoglobulin G $(1: 2,000)$ for $1 \mathrm{~h}$, and the hybridized bands were detected with an enhanced chemiluminescence kit (PerkinElmer Life Sciences, Boston, MA).

\section{Statistical analysis}

The data were presented as the means \pm standard deviation (SD). Data were analyzed using SAS 9.3 statistical software (SAS Institute Inc., Cary, North Carolina, USA). Appropriate parametric (t-test) or nonparametric (Mann-Whitney U or Wilcoxon) statistical tests were used to make comparisons between the 2 groups. In all analyses, $\mathrm{P}<0.05$ was considered to indicate statistical significance.

\section{Results}

\section{Phenol red thread test}

We measured aqueous tear secretion using the cotton thread test. As illustrated in Fig. 1, no significant difference in wetting length was observed between four groups on the baseline $(\mathrm{P}>0.05)$. There was no significant difference between group Con and group Sal at all time points $(\mathrm{P}>0.05)$. In group Fin, the tear secretion markedly decreased after receiving finasteride, with a statistically significant difference compared with that of group Con on days 14, 21 and 28 ( $\mathrm{P}=0.041, \mathrm{P}=0.014$, and $\mathrm{P}=0.014$, respectively). Compared with group Con, the tear secretion of group $B p$ slightly decreased with $B p$ therapy, and the wetness length of the thread of group $B p$ was significantly longer than that of group Fin on days 21 and $28(\mathrm{P}=0.027$ and $\mathrm{P}=0.045$, respectively $)$. 
Fig. 1. Changes in tear secretion in four groups at different time points (unit: $\mathrm{mm}$ ). Note: ${ }^{*} \mathrm{P}<0.05 /{ }^{* *} \mathrm{P}<0.01$ vs. baseline within the same group. $\boldsymbol{\Delta} \mathrm{P}<0.05 / \mathbf{\Delta} \boldsymbol{\Delta} \mathrm{P}<$ 0.01 within the group Con at the same time point. ${ }^{\#} \mathrm{P}<0.05$ within the group Fin at the same time point.

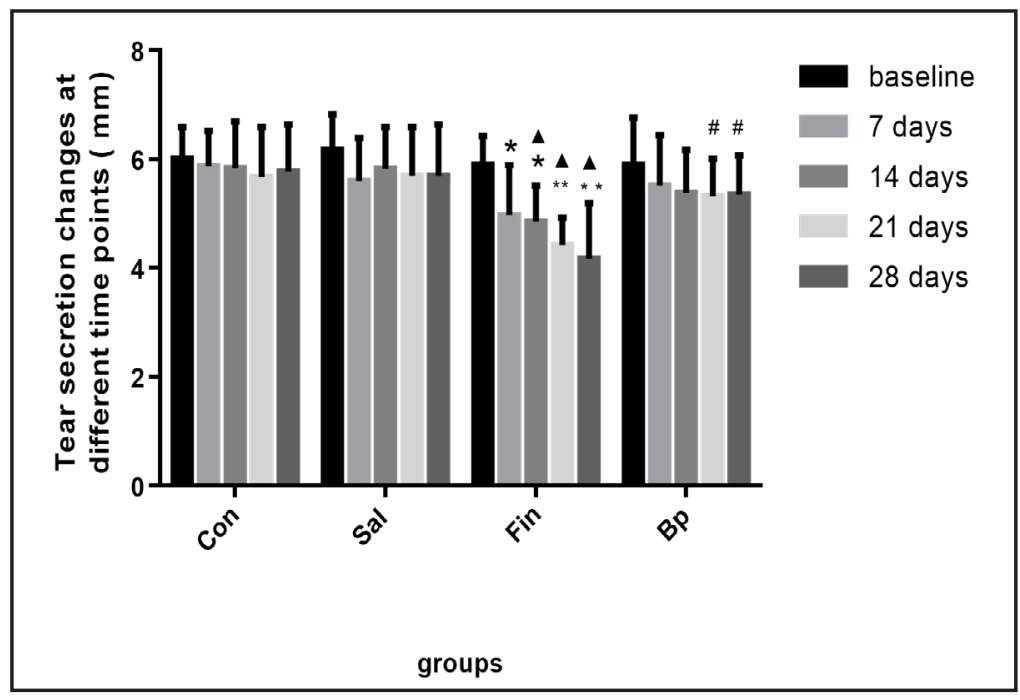

Fig. 2. BUT changes in the four groups at different time points (unit: s). Note: ${ }^{*} \mathrm{P}<$ $0.05, * * \mathrm{P}<0.01$ vs. baseline within the same group. $\mathbf{\Delta} \mathrm{P}$ $<0.05$ within the group Con at the same time point. $\mathrm{AP}<$ 0.05 within the group Fin at the same time point.

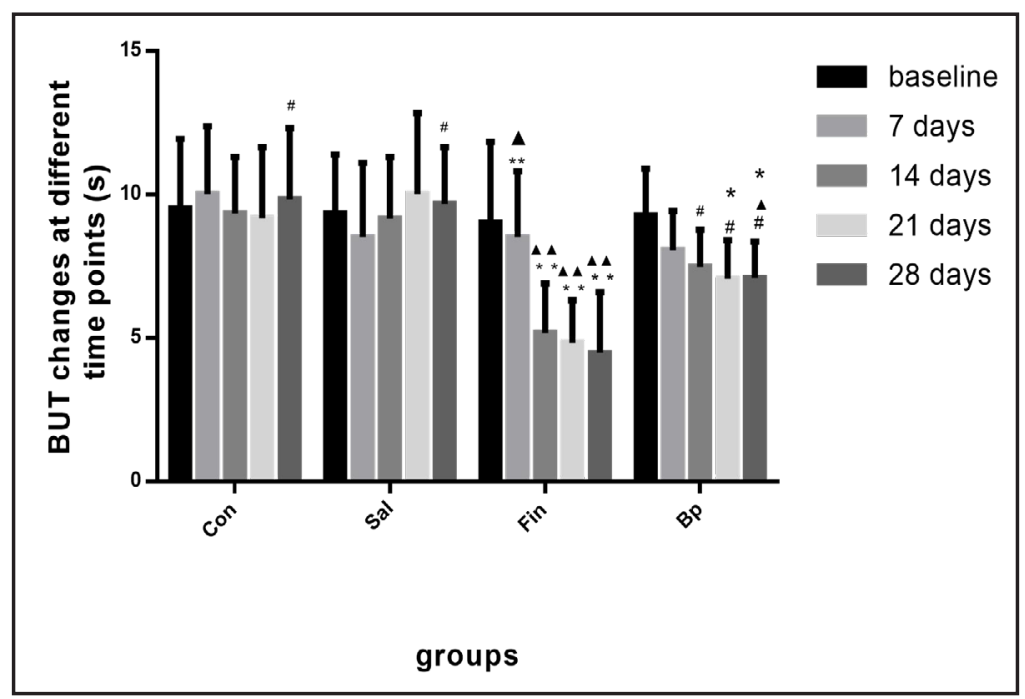

Tear film breakup time (BUT)

As illustrated in Fig. 2, the BUT values of the four groups were not different from the baseline $(\mathrm{P}>0.05)$. There was no significant difference between group Con and group Sal at all time points $(\mathrm{P}>0.05)$. The BUT value of group Fin markedly decreased after receiving finasteride, with significant differences compared with that of group Con on days 7, 14, 21 and $28(\mathrm{P}=0.041, \mathrm{P}=0.003, \mathrm{P}=0.004$, and $\mathrm{P}=0.002$, respectively). The decreased value of BUT in group $B p$ was relatively smaller than that in group Fin, and there were significant differences between those two groups on day 14,21 and $28(\mathrm{P}=0.026, \mathrm{P}=0.021$, and $\mathrm{P}=$ 0.027 , respectively).

\section{Corneal epithelial fluorescein staining}

As illustrated in Fig. 3, there was no corneal punctate staining in any eyes at baseline. No significant difference in scores was noted between group Sal and group Con at all time points $(P>0.05)$. Finasteride induced a significant increase in corneal staining 14 days after administration, and there was a significant difference compared with that of group Con on day 21 and $28(\mathrm{P}=0.005, \mathrm{P}=0.005$, respectively). Meanwhile, the staining scores in group $B p$ were significantly lower than those in group Fin on days 21 and $28(\mathrm{p}=0.037$ and $\mathrm{p}=$ 0.016 , respectively).

\section{KARGER}


Fig. 3. Changes in corneal epithelial fluorescein staining in the four groups at different time points (unit: s). Note: ${ }^{*} \mathrm{P}$ $<0.05 /^{* *} \mathrm{P}<0.01$ vs. baseline within the same group. $\boldsymbol{\Delta} \mathrm{P}$ $<0.05$ within the group Con at the same time point. $\mathrm{AP}<$ 0.05 within the group Fin at the same time point.

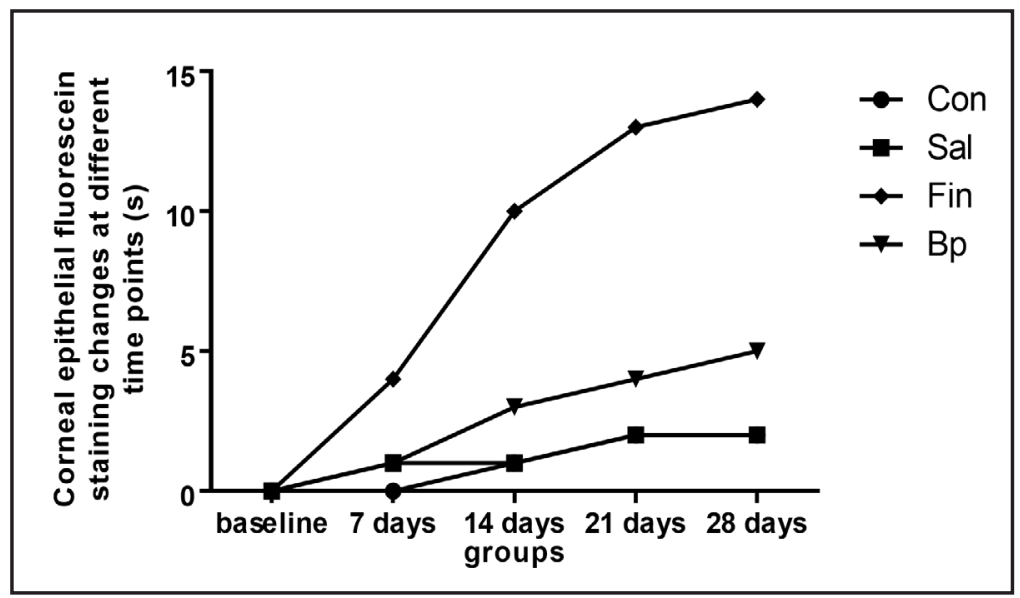

Fig. 4. Serum level of testosterone changes in four groups at different time points (unit: s). Note: ${ }^{*} \mathrm{P}<0.05 /{ }^{* *} \mathrm{P}<0.01$ vs. baseline within the same group. ${ }^{\boldsymbol{\Delta}} \mathrm{P}<0.05 /{ }^{\boldsymbol{\Delta}} \mathrm{P}<0.01$ within the group Con at the same time point.

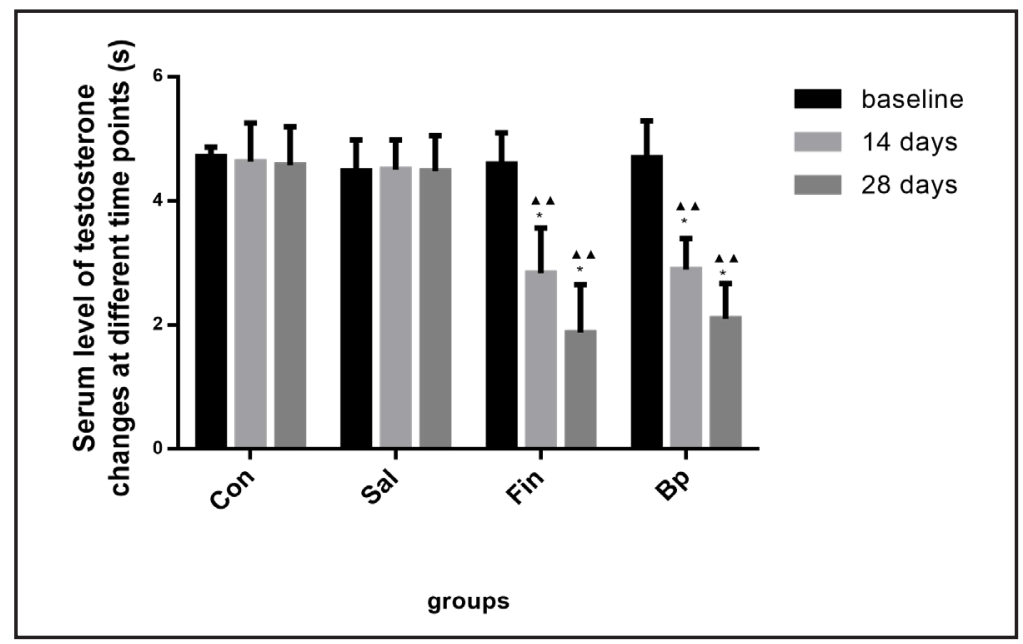

Determination of testosterone (T)

The serum level of testosterone in different groups was measured at baseline, day 14 and day 28, and the results are shown in Fig. 4. The testosterone level of four groups showed no significant differences from baseline $(\mathrm{P}>0.05)$. There was no significant difference between group Con and group Sal at all time points ( $p>0.05)$. In contrast with group Con and group Sal, the levels of testosterone of group Fin and group $B p$ were all decreased on day 14 and $28(\mathrm{p}<0.05)$. No significant difference was identified between group Fin and group $\mathrm{BP}$ at all time points $(\mathrm{P}>0.05)$.

\section{Lacrimal glands and corneal histopathology}

The pathologic changes of lacrimal glands in each group on day 28 are described in Fig. 5 (H\&E staining). Lacrimal glands from group Con contained a well-preserved acinar structure and showed no significant lymphocyte infiltration (Fig. 5A). In group Fin, large quantities of lymphocyte infiltration were shown, and there was a severe inflammatory response, with inflammatory cells invading the interlobular space and surrounding both acinar and ductal cells (Fig. 5B). In group $B p$, there was much less inflammation (Fig. 5C).

Pathologic changes in the corneas of each group on day 28 are described in Fig. 8 (H\&E staining, 10×). The cornea from group Con had a normal appearance in both thickness and cellular morphology (Fig. 6A). In group Fin and group $B p$, the corneas also appeared normal, and no obvious increase in the infiltration of inflammatory cells was observed (Fig. 6B, 6C).

Cytokine assay results

To investigate the changes in inflammatory cytokine expression levels in different groups and to select the relevant candidate genes for further study, gene microarray methods were 


\section{Cellular Physiology Cell Physiol Biochem 2016;39:266-277 \begin{tabular}{l|l} 
DOI: 10.1159/000445622 & and Biochemistry 2016 The Author(s). Published by S. Karger AG, Basel \\
Published online: June 24, 2016 & $\begin{array}{l}\text { www.karger.com/cpb } \\
\text { and }\end{array}$
\end{tabular}

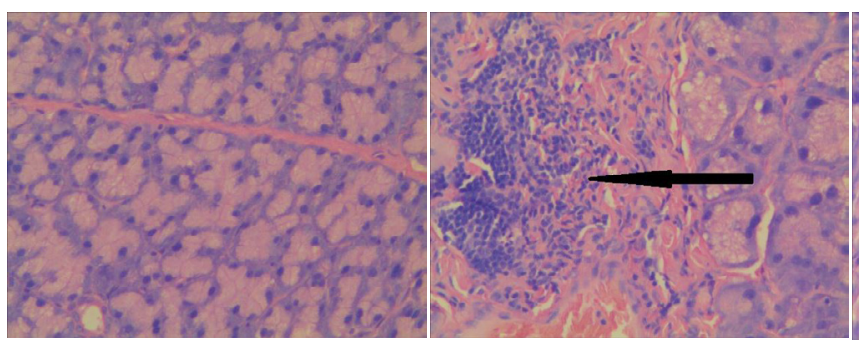

A
B

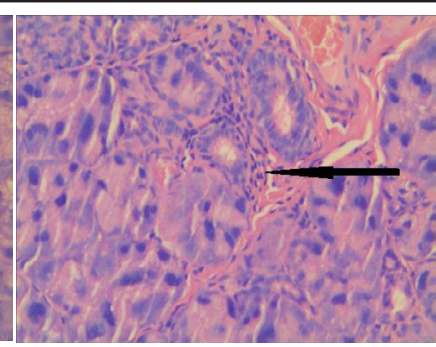

$\mathrm{C}$

Fig. 5. Lacrimal gland histopathology (hematoxylin-eosin staining, $\times 400$ ). (A) Lacrimal gland from group Con; (B) Lacrimal gland from Group Fin; (C) Lacrimal gland from Group Bp.

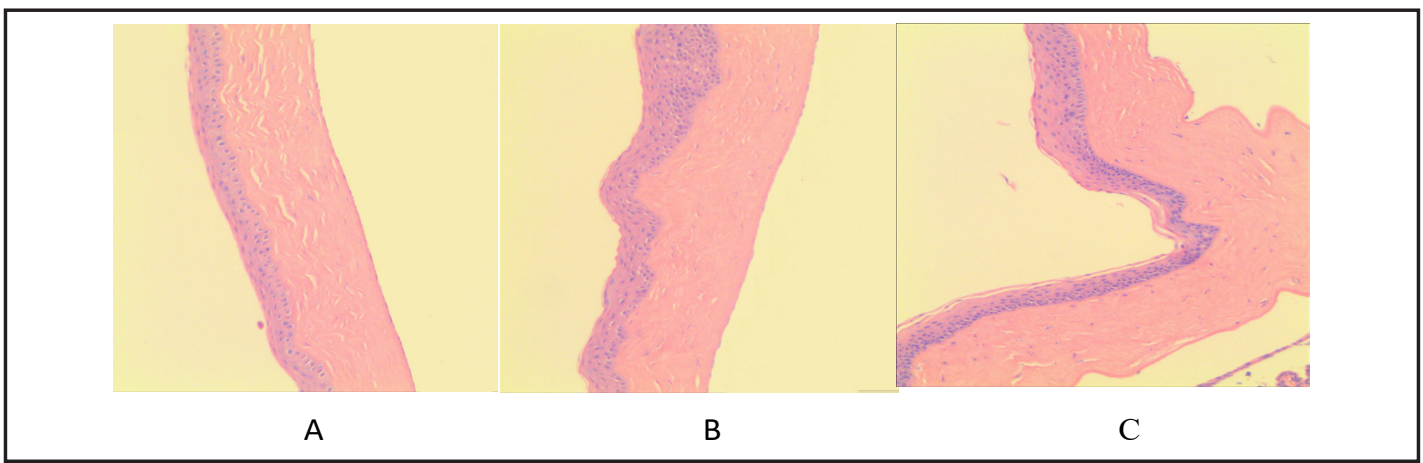

Fig. 6. Cornea histopathology (hematoxylin-eosin staining, $\times 100$ ). (A) Cornea from group Con; (B) Cornea from Group Fin; (C) Cornea from Group Bp.

used to analyze the lacrimal gland and cornea tissues for inflammatory cytokines (Table 2 and Fig. 7).

Expression levels of 22 cytokines in the lacrimal gland were changed significantly in group Fin compared to group Con. Most of these cytokines were considered to be inflammatory factors and chemokines. The expression levels of 15 cytokines, including B7-2/ Cd86, CNTF, Fas L, GM- CSF, ICAM-1, IFN- $\gamma$, IL-1 $\beta$, IL-4, IL-6, IL-10, Leptin, L-Selectin, MMP8 , TIMP-1, and TNF- $\alpha$, were significantly increased. Among the highly expressed cytokines, IL-1 $\beta$, IL-6, GM- CSF, and TNF- $\alpha$ are proinflammatory cytokines, and IL- 4 and IL-10 are antiinflammatory genes. Meanwhile, the expression of those cytokines decreased significantly in group $B p$ compared to group Fin. When compared with group Con, the expression of almost all cytokines in group $B p$ showed no significant differences; only several cytokines were moderately increased.

The expression levels of 5 cytokines in the cornea were significantly elevated in group Fin compared to group Con, including B7-2/Cd86, Fractalkine, IL-13, PDGF-AA, Prolactin $\mathrm{R}$, and TIMP-1. The expression of almost all cytokines in group $B p$ showed no significant differences when compared to group Con.

\section{Western blot}

High expression and inflammatory-related cytokines in the lacrimal gland, including B7-2/Cd86, IL-1 $\beta$, IL-4, IL-6, IL-10, MMP-8, FasL, TNF- $\alpha$ and TIMP-1, were selected as candidates for further Western blot analysis. Their expression levels are shown in Table 3 and Fig. 8. The expression levels of IL-1 $\beta$, IL-4, IL-6, IL-10, MMP-8, FasL, and TNF- $\alpha$ were significantly increased in group Fin compared to group Con $(\mathrm{P}<0.05$ or $\mathrm{P}<0.01)$. B7-2/Cd86 and TIMP-1 were also increased with no statistical significance.

Compared with group Fin, IL-1 $\beta$, FasL, and TNF- $\alpha$ in group $B p$ were significantly decreased $(P<0.05)$. Other cytokines also showed this decreased tendency but without statistical significance. 
Table 2. Results of the cytokine assay in the lacrimal gland and cornea of three groups compared with the control group

\begin{tabular}{|c|c|c|c|c|c|c|}
\hline \multirow[t]{2}{*}{ Name of cytokine } & \multicolumn{2}{|c|}{ Folds of group Fin to Con } & \multicolumn{2}{|c|}{ Folds of group $B p$ to Con } & \multicolumn{2}{|c|}{ Folds of group $B p$ to Fin } \\
\hline & $\begin{array}{l}\text { Lacrimal } \\
\text { gland }\end{array}$ & corneal & $\begin{array}{l}\text { Lacrimal } \\
\text { gland }\end{array}$ & corneal & $\begin{array}{l}\text { Lacrimal } \\
\text { gland }\end{array}$ & corneal \\
\hline \multicolumn{7}{|l|}{ Inflammatory cytokines } \\
\hline IL-1 $\alpha$ & 1.220 & 1.080 & 1.191 & 1.018 & 0.812 & 0.971 \\
\hline IL-1 $\beta$ & $1.533 \uparrow$ & 1.027 & 0.990 & 1.015 & $0.707 \downarrow$ & 0.930 \\
\hline IL-2 & 1.283 & 1.040 & 1.004 & 0.962 & $0.752 \downarrow$ & 0.958 \\
\hline IL-6 & $1.357 \uparrow$ & 1.145 & 0.951 & 1.218 & $0.689 \downarrow$ & 1.094 \\
\hline IFN- $\gamma$ & $1.827 \uparrow$ & 1.063 & 1.125 & 0.801 & $0.675 \downarrow$ & 1.029 \\
\hline Fas Ligand & $3.096 \uparrow$ & $1.357 \uparrow$ & 0.772 & 1.168 & $0.544 \downarrow$ & 0.912 \\
\hline GM- CSF & $1.952 \uparrow$ & 0.941 & 0.867 & 0.908 & $0.659 \downarrow$ & 1.092 \\
\hline TNF- $\alpha$ & $1.541 \uparrow$ & 0.876 & $0.721 \downarrow$ & 1.200 & $0.738 \downarrow$ & 1.148 \\
\hline \multicolumn{7}{|c|}{ Anti-inflammatory cytokines } \\
\hline IL-1 R6 & $1.334 \uparrow$ & 0.854 & 0.949 & 0.875 & $0.750 \downarrow$ & 1.090 \\
\hline IL-4 & $1.716 \uparrow$ & 1.123 & 0.857 & 1.296 & $0.573 \downarrow$ & 1.053 \\
\hline IL-10 & $1.593 \uparrow$ & 1.256 & 0.816 & 1.144 & $0.642 \downarrow$ & 0.898 \\
\hline IL-13 & $1.435 \uparrow$ & $1.427 \uparrow$ & 0.904 & 1.191 & 0.817 & 0.981 \\
\hline \multicolumn{7}{|l|}{ Chemokines } \\
\hline MCP-1 & $1.469 \uparrow$ & 0.996 & 0.926 & 1.106 & 0.834 & 1.071 \\
\hline MIP-3 $\alpha$ & $1.375 \uparrow$ & 1.022 & $1.306 \uparrow$ & 1.155 & 0.865 & 1.009 \\
\hline Fractalkine & 1.194 & 0.971 & 1.198 & 0.910 & 0.882 & $1.352 \uparrow$ \\
\hline CINC-1 & 1.136 & 1.010 & 0.995 & 1.008 & 0.876 & 0.998 \\
\hline CINC- $2 \alpha$ & 1.216 & 1.221 & 0.928 & 1.047 & $0.763 \downarrow$ & 0.857 \\
\hline CINC-3 & 1.229 & 1.224 & 0.983 & 1.001 & 0.800 & 0.818 \\
\hline Thymus Chemokine- 1 & 1.085 & 1.182 & 1.125 & 1.069 & 0.963 & 0.920 \\
\hline \multicolumn{7}{|l|}{ Growth factors } \\
\hline$\beta-N G F$ & $1.448 \uparrow$ & 0.932 & $1.441 \uparrow$ & 0.893 & $0.725 \downarrow$ & 1.130 \\
\hline PDGF- AA & $1.330 \uparrow$ & $1.310 \uparrow$ & $1.323 \uparrow$ & 1.195 & 0.817 & 0.754 \\
\hline VEGF & 1.291 & 1.078 & 1.286 & 0.922 & 0.839 & 0.869 \\
\hline CNTF & $1.809 \uparrow$ & 1.161 & 0.872 & 1.212 & $0.595 \downarrow$ & 0.874 \\
\hline Activin A & $1.343 \uparrow$ & 0.963 & 1.081 & 1.069 & 0.805 & 1.110 \\
\hline \multicolumn{7}{|l|}{ Other cytokines } \\
\hline $\mathrm{B} 7-2 / \mathrm{Cd} 86$ & $1.992 \uparrow$ & $1.478 \uparrow$ & 1.176 & 1.255 & $0.590 \downarrow$ & 0.849 \\
\hline Agrin & 1.054 & 0.963 & 1.034 & 0.932 & 0.950 & 1.048 \\
\hline Leptin & $2.315 \uparrow$ & 1.122 & 0.848 & $1.657 \uparrow$ & $0.659 \downarrow$ & 1.050 \\
\hline MMP-8 & $3.304 \uparrow$ & 1.128 & $0.640 \downarrow$ & 1.225 & $0.623 \downarrow$ & $0.738 \downarrow$ \\
\hline TIMP-1 & $1.528 \uparrow$ & $1.336 \uparrow$ & 1.045 & $1.304 \uparrow$ & $0.760 \downarrow$ & $0.765 \downarrow$ \\
\hline LIX & 1.295 & 1.028 & 1.246 & 0.953 & 0.884 & 0.972 \\
\hline L-Selectin & $1.664 \uparrow$ & 0.998 & 0.926 & 1.067 & 0.814 & 1.059 \\
\hline Prolactin R & 1.232 & $1.305 \uparrow$ & 1.240 & 1.271 & 0.860 & 0.780 \\
\hline RAGE & 1.116 & 0.952 & 1.111 & 1.031 & 0.926 & 1.076 \\
\hline ICAM-1 & $1.862 \uparrow$ & 0.944 & 0.982 & $0.713 \downarrow$ & 0.771 & 1.059 \\
\hline
\end{tabular}

\section{Discussion}

A few studies have reported the impact of dry eye on vision-related quality of life [16]. Currently, the treatment for dry eye disease is largely palliative, as the underlying mechanisms of this sight-compromising syndrome are not yet fully understood [17]. It is prudent to explore the pathogenesis and therapy of dry eye. Recent findings from human beings and animal models indicate that an inflammatory response exists in the lacrimal gland and may 


\section{Cellular Physiology Cell Physiol Biochem 2016;39:266-277

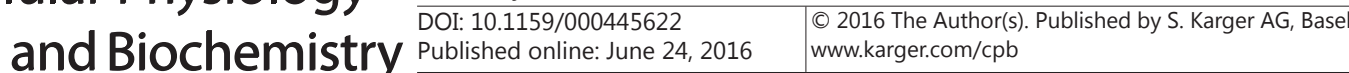

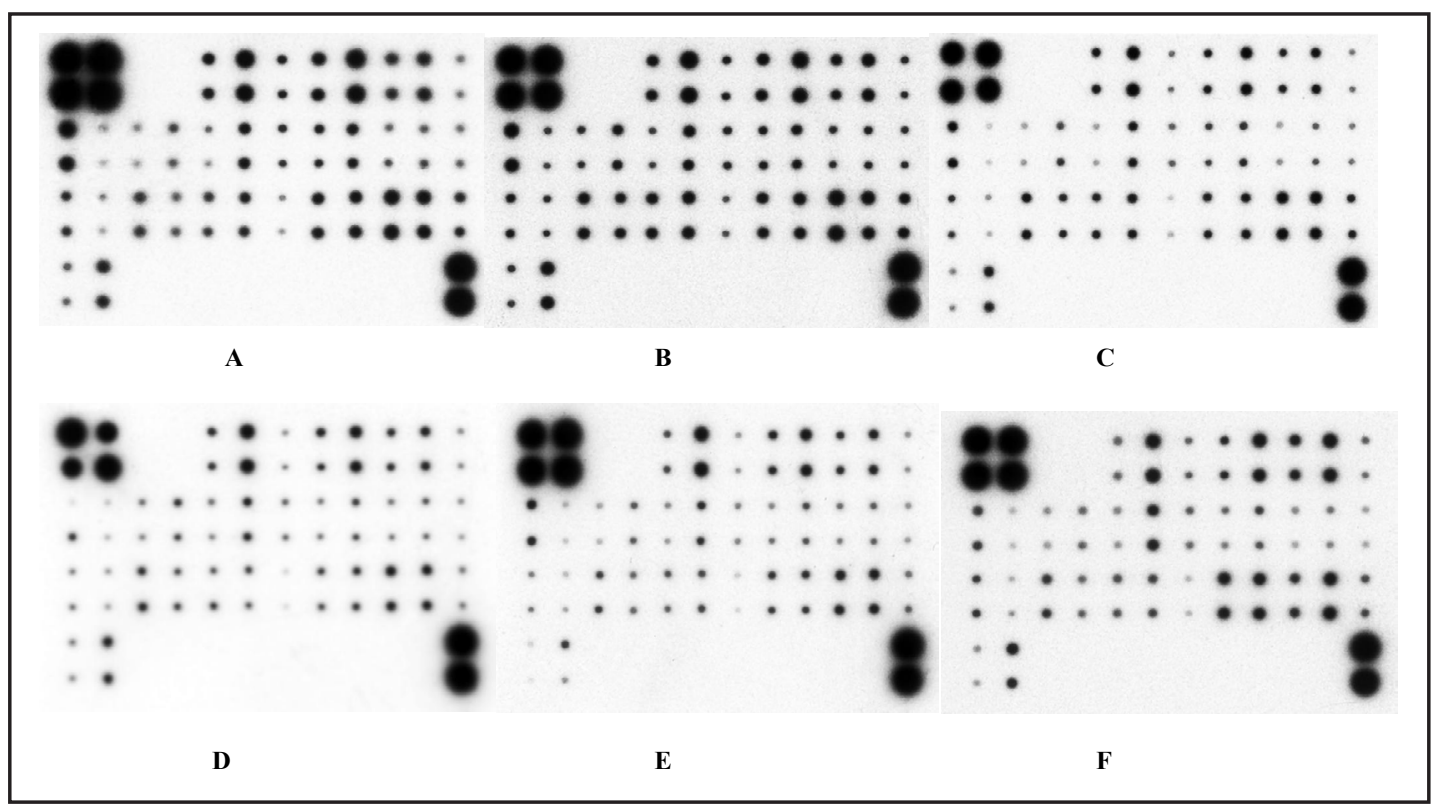

Fig. 7. Microarray analysis of inflammatory factors secreted from the lacrimal gland and cornea. (A) lacrimal gland of group Con; (B) lacrimal gland of group Fin; (C) lacrimal gland of group Bp; (D) cornea of group Con; (E) cornea of group Fin; (F) cornea of group Bp.

Fig. 8. Western blot analysis of secreted inflammatory factors from the lacrimal gland. (A) group Con; (B) Group Fin; (C) Group Bp.

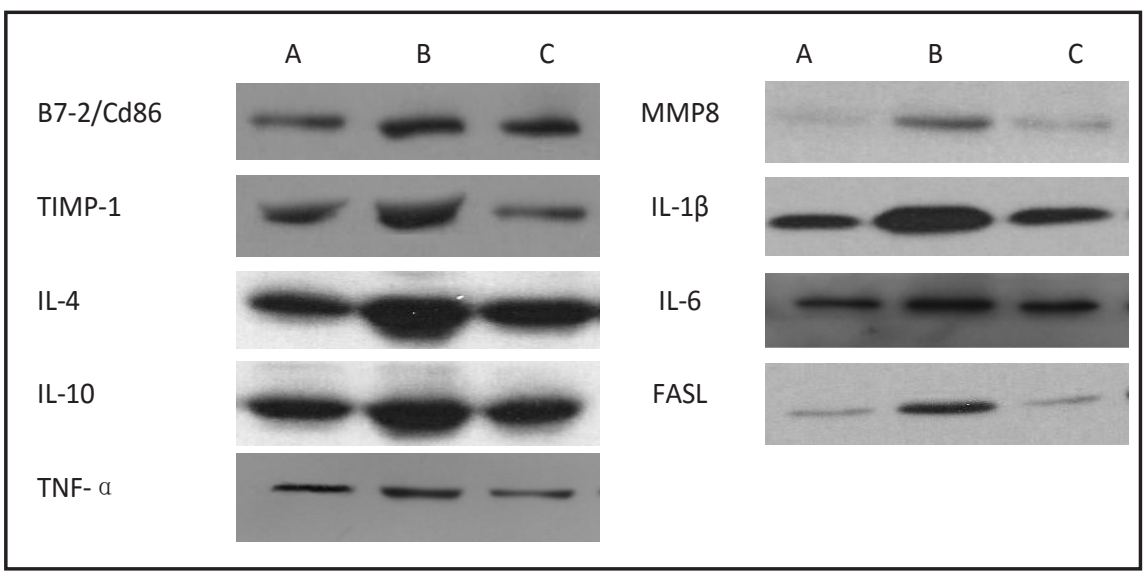

Table 3. Western blot analysis of cytokines in the lacrimal gland of the three groups of rats. Note: ${ }^{*} \mathrm{P}<$ $0.05 /{ }^{* *} \mathrm{P}<0.01$ within the group Con. $\mathbf{\Delta} \mathrm{P}<0.05 / \mathbf{\Delta} \mathbf{\Delta} \mathrm{P}<0.01$ within the group Fin

\begin{tabular}{|c|c|c|c|c|c|c|c|c|c|}
\hline group & $\mathrm{B} 7-2 / \mathrm{Cd} 86$ & MMP8 & TIMP-1 & $\mathrm{IL}-1 \beta$ & II-4 & $\overline{\text { IIL-6 }}$ & IL-10 & $\overline{\text { FASL }}$ & $\overline{\text { TNF- } a}$ \\
\hline con & $0.602 \pm 0.192$ & $0.479 \pm 0.239$ & $0.740 \pm 0.302$ & $0.718 \pm 0.306$ & $0.580 \pm 0.172$ & $0.536 \pm 0.143$ & $0.831 \pm 0.199$ & $0.556 \pm 0.238$ & $0.507 \pm 0.099$ \\
\hline Fin & $0.872 \pm 0.504$ & $0.906 \pm 0.239 * *$ & $1.091 \pm 0.347$ & $1.112 \pm 0.234 *$ & $1.016 \pm 0.333 *$ & $1.021 \pm 0.425 *$ & $1.261 \pm 0.295 *$ & $0.976 \pm 0.293 *$ & $0.893 \pm 0.387 *$ \\
\hline$B P$ & $0.791 \pm 0.704$ & $0.810 \pm 0.276 *$ & $0.750 \pm 0.506$ & $0.661 \pm 0.310 \Lambda$ & $0.804 \pm 0.272$ & $0.858 \pm 0.322$ & $0.908 \pm 0.315$ & $0.461 \pm 0.466 \boldsymbol{\Lambda}$ & $0.472 \pm 0.117 \boldsymbol{\Lambda}$ \\
\hline
\end{tabular}

significantly contribute to the pathophysiology of dry eye. Currently, the main therapies for dry eye mainly focus on relieving symptoms but do not aim to block the pathogenesis of dry eye. Although cyclosporine and steroid hormone were developed for the treatment of dry eye by inhibiting the immune response, they can induce some inevitable side effects, such as the impairment of the ocular surface defense barrier and secondary infection [18]. At present, there have been few studies on traditional Chinese medicine interventions for dry eye. In

\section{KARGER}




\section{Cellular Physiology Cell Physiol Biochem 2016;39:266-277 \\ and Biochemistry Published online: June 24, $2016 \quad \begin{aligned} & \text { DOI: 10.1159/000445622 } 2016 \text { The Author(s). Published by S. Karger AG, Basel } \\ & \text { www.karger.com/cpb }\end{aligned}$ \\ Zhang et al.: The Effect of Bidens Pilosa L. on Dry Eye}

our previous studies $[13,14], B p$ extracts were demonstrated to have anti-inflammatory activities by inhibiting the production of pro-inflammatory cytokines. Furthermore, no obvious side effects were observed after the long-term oral administration of $B p$, which is consistent with another study on the acute toxicity of BP in rats [19]. Therefore, $B p$ seems to be a promising therapeutic candidate for dry eye.

Androgen deficiency rats develop dry eye, which is characterized by decreased tear secretion, ocular surface damage and lacrimal gland inflammation, and it is an animal model used in the study of dry eye [20]. In the present study, the level of serum testosterone decreased significantly after finasteride administration; we noted that finasteride administration showed a significant reduction in the tear flow and a severe inflammatory response in the lacrimal gland. The tear secretion in group Fin was significantly lower than group Con on days 14, 21, and 28, and the level of BUT in group Fin was significantly lower than group Con on days $7,14,21$, and 28. In addition, the corneal fluorescein staining scores in group Fin were significantly higher than in group Con on day 21 and 28. Therefore, direct finasteride administration in normal rats could produce a suitable model for sex steroid-deficient dry eye in rats. This model can be used to study the effect of topically applied pharmacological agents for androgen deficiency dry eye. In the current study, the female rats were selected according to the high incidence rate of women in the clinic. The decline of androgen levels is a main cause of dry eye. The condition is more prevalent in women and increases with age because of the decline of androgen level with age, especially after menopause. The results obtained by Singh also found that finasteride administration in both sexes of normal rats could produce a suitable model for sex steroid-deficient dry eye in rats, but finasteride administration significantly downregulated androgen receptors in the lacrimal gland of the female rats by 8 times compared to male rats. Correspondingly, finasteride-treated female rats showed a $49 \%$ reduction of tear flow, while male rats showed a $40 \%$ reduction tear flow.

Approximately 200 different compounds have been discovered in $B p$, and their structures have been well elucidated. Among them, the main compounds are flavonoids and polyacetylenes, which are major anti-inflammatory phytochemicals in $B p[21,22]$. In addition, both androgen and flavonoids are polyphenolic heterocyclic compounds. It was reported that flavonoid compounds have androgen-like effects [23] and can be used to treat certain diseases due to the decrease of androgen levels, such as osteopenia [24]. Considering that androgen receptor (AR) is widely present in the lacrimal gland, cornea and other ocular tissues [25], $B p$ should also produce androgen-like effects to treat dry eye, a disease that mainly results from the decrease of the androgen level.

Most of the patients visit the clinic after the development of dry eye, so the major concern of the present study was to investigate the therapeutic effect of $B p$ on dry eye symptoms. However, herbal medicines, such as BP, usually exert their curative effect after long-term administration. Considering that the finasteride-treated rat is a well-validated dry eye model, $B p$ and finasteride were co-administered to the rats in our study. In the present study, we investigated the effect of $B p$ treatment for 28 days on the tear functions and corneal epithelial status in androgen-deficient rats. We observed that tear secretion, tear film stability, and corneal epithelial staining were improved significantly with $B p$ treatment. $B p$ has also been shown to alleviate inflammatory cell infiltration in the lacrimal gland.

To determine which cytokines are involved in the pathogenesis of dry eye and the curative effect of $B p$, cytokine antibody array analysis was performed, which was further verified by western blot analysis. Gene expression profiling technologies allow for large panels of genes to be analyzed at one time; therefore, a large quantity of information on cytokine gene expression levels can be collected throughout the course of inflammatory diseases [26]. Such arrays could be informative in the ongoing study of the role of cytokines in disease pathogenesis and treatment. In the present study, 35 different cytokine and cytokine-related proteins were screened from the rat lacrimal gland and cornea in different groups. Protein expression assays were used to determine whether cytokine and cytokinerelated protein levels in the lacrimal gland or cornea were increased or decreased and whether such changes were associated with tissue histopathology and clinical presentation. 


\section{Cellular Physiology Cell Physiol Biochem 2016;39:266-277 \begin{tabular}{l|l} 
and Biochemist 10.1159/000445622 & $\begin{array}{l}\text { D } 2016 \text { The Author(s). Published by S. Karger AG, Basel } \\
\text { www.karger.com/cpb }\end{array}$ \\
\hline Published online: June 24, 2016
\end{tabular} \\ Zhang et al.: The Effect of Bidens Pilosa L. on Dry Eye}

Our results showed that the lack of testosterone could influence the cytokine expression in the lacrimal gland. From the results of antibody array analysis, we observed that 22 cytokines in the lacrimal gland were changed significantly in group Fin compared to group Con. The expression levels of 15 cytokines were significantly increased, most of which were inflammatory factors and chemokines. Specifically, the expression levels of those cytokines decreased with $B p$ treatment. Inflammation-related cytokines highly expressed in the lacrimal gland were selected as the candidates for further Western blots analysis. The results showed the expression levels of IL-1 $\beta$, IL-4, IL-6, IL-10, MMP-8, FasL and TNF- $\alpha$ significantly increased in group Fin compared to group Con. Meanwhile, IL-1 $\beta$, FasL, and TNF- $\alpha$ were significantly decreased after $B p$ administration. In the present study, the expression levels of pro-inflammatory cytokines, including IL- $1 \beta$, IL-6, GM-CSF, and TNF- $\alpha$, in group Fin were upregulated compared with group Con; meanwhile, the levels of anti-inflammatory cytokines, such as IL-4 and IL-10, in group Fin were also upregulated compared with those in group Con. The above data suggested that the inflammation in androgen deficiency dry eye resulted from the imbalance between inflammatory responses and anti-inflammatory responses. Whether the inflammatory response appeared in the cornea was also explored in this study. Our findings indicated that the levels of inflammatory cytokines were not increased in the cornea, which was consistent with corneal histopathology.

In conclusion, our study demonstrated that severe inflammation occurred in the lacrimal gland in androgen deficiency dry eye, suggesting that a correlation between sex steroid deficiency and the inflammatory response may exist. The long-term administration of $B p$ could alleviate the dry-eye symptoms, including lacrimal gland inflammation, tear secretion and the stability of the tear film. As finasteride administration caused 16-25\% tear reduction and did not develop into moderate to severe dry eye, we only observed the efficacy of $B p$ in a mild dry eye condition. The efficacy of $B p$ on different degrees of dry eye should be further investigated. Although further studies of the mechanisms are still necessary, our findings clearly suggest that $B p$ may be further developed as a therapeutic agent to treat androgen deficiency dry eye.

\section{Acknowledgements}

This work was supported by Jiangsu Provincial Natural Science Fund of China (No. BK20141504) and the Six Talent Peaks Project in Jiangsu Province (No. WSW-048).

\section{Disclosure Statement}

None of the authors have any conflicts of interest related to this paper.

\section{References}

1 Smith RE: The tear film complex: pathogenesis and emerging therapies for dry eyes. Cornea 2005;24:1-7.

2 Lemp MA: Report of the National Eye Institute/Industry workshop on Clinical Trials in Dry Eyes. CLAO J 1995;21:221-232.

3 Lee WB, Hamilton SM, Harris JP, Schwab IR: Ocular complications of hypovitaminosis a after bariatric surgery. Ophthalmology 2005;112:1031-1034.

4 No authors listed: The epidemiology of dry eye disease: report of the Epidemiology Subcommittee of the International Dry Eye WorkShop (2007). Ocul Surf 2007;5:93-107.

5 Schaumberg DA, Sullivan DA, Buring JE, Dana MR: Prevalence of dry eye syndrome among US women. Am J Ophthalmol 2003;136:318-326.

6 Serrander AM, Peek KE: Changes in contact lens comfort related to the menstrual cycle and menopause. A review of articles. J Am Optom Assoc 1993;64:162-166. 


\section{Cellular Physiology Cell Physiol Biochem 2016;39:266-277

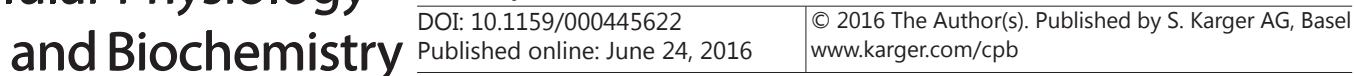 \\ Zhang et al.: The Effect of Bidens Pilosa L. on Dry Eye}

7 Silva FL, Fischer DC, Tavares JF, Silva MS, de Athayde-Filho PF, Barbosa-Filho JM: Compilation of secondary metabolites from Bidens pilosa L. Molecules 2011;16:1070-1102.

8 Horiuchi M, Seyama Y: Improvement of the Antiinflammatory and Antiallergic Activity of Bidens pilosa L. var. radiata SCHERFF Treated with Enzyme (Cellulosine). J Health Science 2008;54:294-301.

9 Alvarez A, Pomar F, Sevilla, Montero MJ: Gastric antisecretory and antiulcer activities of an ethanolic extract of Bidens pilosa L. var. radiata Schult. Bip. J Ethnopharmacol 1999;67:333-340.

10 Pereira RL, Ibrahim T, Lucchetti L, da Silva AJ, Goncalves de Moraes VL: Immunosuppressive and anti-inflammatory effects of methanolic extract and the polyacetylene isolated from Bidens pilosa L. Immunopharmacology 1999;43:31-37.

11 Chang SL, Chiang YM, Chang CL, Yeh HH, Shyur LF, Kuo YH, Wu TK, Yang WC: Flavonoids, centaurein and centaureidin, from Bidens pilosa, stimulate IFN-gamma expression. J Ethnopharmacol 2007;112:232-236.

12 Chiang YM, Chang CL, Chang SL, Yang WC, Shyur LF: Cytopiloyne, a novel polyacetylenic glucoside from Bidens pilosa, functions as a T helper cell modulator. J Ethnopharmacol 2007;110:532-538.

13 Li K, Wang Y, Zhang C: Influence of bidens bipinnata l on tear secretion and tear film stability of dry eye model, and expression of proinflammatory cytokines and apoptosis related factors in lacrimal gland. China J Chinese Ophthalmol 2013;23:389-392.

14 Li K, Wang YL, Gao WP, Wang T, Wang YF: Effect of runmuling on aqueous tear deficiency xeroma. Int J Ophthalmol 2009;11:2116-2117.

15 Frida L, Rakotonirina S, Rakotonirina A, Savineau JP: In vivo and in vitro effects of Bidens pilosa L. (Asteraceae) leaf aqueous and ethanol extracts on primed-oestrogenized rat uterine muscle. Afr J Tradit Complement Altern Med 2007;5:79-91.

16 Tong L, Waduthantri S, Wong TY, Saw SM, Wang JJ, Rosman M, Lamoureux E: Impact of symptomatic dry eye on vision-related daily activities: the Singapore Malay Eye Study. Eye (Lond) 2010;24:1486-1491.

17 Khajavi N, Reinach PS, Skrzypski M, Lude A, Mergler S: L-carnitine reduces in human conjunctival epithelial cells hypertonic-induced shrinkage through interacting with TRPV1 channels. Cell Physiol Biochem 2014;34:790-803.

18 Ma X, Zou J, He L, Zhang Y: Dry eye management in a Sjogren's syndrome mouse model by inhibition of p38-MAPK pathway. Diagn Pathol 2014;9:5.

19 Shen AZ, Li X, Hu W, Chen FH: Total flavonoids of Bidens bipinnata L. ameliorate experimental adjuvantinduced arthritis through induction of synovial apoptosis. BMC Complement Altern Med 2015;15:437.

20 Singh S, Moksha L, Sharma N, Titiyal JS, Biswas NR, Velpandian T: Development and evaluation of animal models for sex steroid deficient dry eye. J Pharmacol Toxicol Methods 2014;70:29-34.

21 Cortés-Rojas D, Chagas-Paula D, Fernando DC, Souza C, Oliveira W: Bioactive compounds in Bidens pilosa L. populations: A key step in the standardization of phytopharmaceutical preparations. Braz. J. Pharmacog 2013;23:28-35.

22 Tan PV, Dimo T, Dongo E: Effects of methanol, cyclohexane and methylene chloride extracts of Bidens pilosa on various gastric ulcer models in rats. J Ethnopharmacol 2000;73:415-421.

23 X H, Y Z, W W: Development of pharmacological research of herba epimedii flavanoid. Chinese Traditional Patent Medicine 2005;27:719-721.

24 Moyad MA: Complementary therapies for reducing the risk of osteoporosis in patients receiving luteinizing hormone-releasing hormone treatment/orchiectomy for prostate cancer: a review and assessment of the need for more research. Urology 2002;59:34-40.

25 Wickham LA, Gao J, Toda I, Rocha EM, Ono M, Sullivan DA: Identification of androgen, estrogen and progesterone receptor mRNAs in the eye. Acta Ophthalmol Scand 2000;78:146-153.

26 Riemer C, Neidhold S, Burwinkel M, Schwarz A, Schultz J, Kratzschmar J, Monning U, Baier M: Gene expression profiling of scrapie-infected brain tissue. Biochem Biophys Res Commun 2004;323:556-564. 